Contents List available at VOLKSON PRESS

Economics \& Management Innovations(EMI)

DOI : http://doi.org/10.26480/icemi.01.2017.423.426

\title{
The Optimum Portfolio of ASEAN Stock Markets: Markov Switching Multivariate Copula Approach
}

\author{
Roengchai Tansuchat ${ }^{1, *}$ \\ 1 Puey Ungphakorn Centre of Excellence in Econometrics, Faculty of Economics,Chiang Mai University, Thailand \\ *Corr. author email: roengchaitan@gmail.com
}

This is an open access article distributed under the Creative Commons Attribution License, which permits unrestricted use, distribution, and reproduction in any medium, provided the original work is properly cited.

\section{ARTICLE DETAILS}

\section{Article History:}

Received 02 october 2017 Accepted 06 october 2017

Available online 11 october 2017

\section{Keywords:}

Optimum Portfolio, ASEAN Stock Markets, Multivariate Copula, Markov Switching Model, Value-atRisk, Expected Shortfall.

\section{ABSTRACT}

The economy of the developing countries in ASEAN (Developing ASEAN) was an international group that had been classified as interesting market for many investors especially when the Western countries experienced economic problems. The research question is how should investors allocate their investment in ASEAN+3 stock index under different situation? The objective of this paper is to construct the optimum portfolio of ASEAN Stock Markets by using Markov Switching Multivariate Gaussian Copula approach based on EGARCH model. The data comprised of stock price index returns from 8 major countries in ASEAN+3 namely, Malaysia, Thailand, Vietnam, Singapore, Indonesia, South Korea, Japan, and China. The data period was from Jan 1, 2007 - May 30,2017. The result showed that ARMA-EGARCH with t-distribution model is fit to model the conditional volatility of stock return in every stock market. Second, the multivariate Gaussian Copula with regime switching were estimated. Finally, the optimal portfolio of each regime was estimated and suggested the optimum shares for each stock market. The Efficient frontier from each portfolio was drawn.

\section{Introduction}

The overall stock markets in the ASEAN region have formed a relatively long average of over 50 years, with some of the stock exchange, such as the Philippines. Indonesia and has been established since 1912 and 1977 respectively, prior to the development of a trading system that continues to the present. While some markets such as Malaysia, Singapore and Thailand have formed later in the same year in 1930, 1962 and 1999, respectively, prior to the establishment of a stock exchange in Ho Chi Minh of Vietnam in 2000, after Vietnam started policy to push the country into an inter-national trade and investment countries.

After Asian financial crisis in 1997, the five stock markets in the original Association of Southeast Asian Nations countries (ASEAN-5), namely Indonesia, Malaysia, the Philippines, Singapore, and Thailand, have more corroboration in order to mitigate that crisis. Consequently, Click and Plummer [1] found that ASEAN-5 stock markets have long run relationship with common trend or cointegration. In the other word, the ASEAN-5 stock markets were integrated in the economic sense, but that integration was far from complete [1]. Later, Karim and Karim [2] found that the stock markets in the ASEAN region were integrated during the pre-, post-1997 and post U.S. subprime financial crisis by using Autoregressive Distributed Lag (ARDL). They also confirmed that the benefits from international portfolio diversification tend to diminish in the long-run term [2].

The economy of the developing countries in ASEAN (Developing ASEAN) was an international group that had been classified as interesting market for many investors especially when the Western countries experienced economic problems. In 2015 the market capitalization of ASEAN stock markets (Thailand, Malaysia, Indonesia, Philippines, Vietnam, and Singapore) was 2.015 trillion US\$ and accounted for 3.26 of world market capitalization. In addition, in 2016 the ASEAN market capitalization expanded $7.41 \%$ comparing to 2015 [3]. Moreover, in case of ASEAN +3 , which is the cooperation between member Association of Southeast Asian Nations (ASEAN) and other 3 countries outside ASEAN namely, Japan, South Korea, and Japan, the market capitalization was jump to 16.33 trillion US\$ and accounted for 26.4 of world market capitalization in 2015. However, the market capitalization of ASEAN +3 recoiled $3.88 \%$ to 16.7 in 2016.
In the literature, there are some studies about optimum portfolio construction by using the dependence from copula approach such as Deng et al. [4] studied portfolio optimization via pair copula-GARCH-EVT-CVaR model, Aussenegg and Cech [5] studied high-dimensional real-world portfolio with new copula approach, Autchariyapanitkuly et al. [6] studied portfolio optimization of stock returns in high-dimensions by using a copula-based approach, and Zhu et al. [7] studied multi-asset portfolio returns by using a Markov switching copula-based approach.

The objective of this paper is to construct the optimum portfolio of ASEAN +3 Stock Markets by using EGARCH with $t$ distribution model and Markov Switching Multivariate Copula. The structure of the remainder of the paper is as follows. Section 2 discusses the econometric models. Section 3 describes the data, descriptive statistics, and unit root test. Section 4 analyzes the empirical estimates from empirical modeling. Some concluding remarks are given in Section 5.

\section{Econometric Models}

\subsection{ARMA(p,q)-GARCH models for univariate distributions}

To model the marginal distribution of each random variable, we employ $\operatorname{ARMA}(p, q)$-Exponential $\operatorname{GARCH}(m, n)$ specification of Nelson $[8,9]$ that can be described both volaility colustering and asymmetry. The EGARCH is given as:

$$
\begin{aligned}
& y_{t}=\phi_{0}+\sum_{i=1}^{p} \phi_{i} y_{t-i}+\sum_{j=1}^{q} \theta_{j} \varepsilon_{t-j}+\varepsilon_{i} \\
& \varepsilon_{t}=h_{t} \eta_{t} \\
& \log h_{t}=\omega+\sum_{i=1}^{m} \alpha_{i}\left|\eta_{t-i}\right|+\gamma \eta_{t-i}+\sum_{j=1}^{n} \beta_{j} h_{t-j}
\end{aligned}
$$

where (1) and (3) are the conditional mean and variance equation, respectively. $\varepsilon_{t}$ is the residual term which consists of the standard variance, $h_{t}$, and the standardized residual, $\eta_{t}$, given by $\eta_{t}=\varepsilon_{t} / \sqrt{h_{t}}$, and is proposed to have a Gaussian distribution or Student- $t$ distribution. 
Asymmetry exists if $\gamma \neq 0$, while leverage exists if $\gamma<0$ and $\gamma<\alpha<-\gamma$. The best-fit $\operatorname{ARMA}(p, q)-\operatorname{EGARCH}(1,1)$ will give the standardized residuals to be transformed into a uniform distribution in $(0,1)$.

\subsection{Sklar's Theorm in $n$-dimensions}

In case of multivariate random variable with $n$ random variables or dimensions, let $H$ is an $n$-dimensional distribution function with margin $F_{1}\left(x_{1}\right), F_{2}\left(x_{2}\right), \ldots, F_{n}\left(x_{n}\right)$. Then there exists an $n$-copula $C$ such that for all $\mathbf{X}$ in $n$ dimensions,

$$
H\left(x_{1}, \ldots, x_{n}\right)=C\left(F_{1}\left(x_{1}\right), \ldots, F_{n}\left(x_{n}\right)\right)=C\left(u_{1}, \ldots, u_{n}\right),
$$

where the marginal distributions, $u_{1}, \ldots, u_{n}$, are uniform in the $[0,1]$ interval. If margins $F_{1}\left(x_{1}\right), \ldots, F_{n}\left(x_{n}\right)$ are all continuous, then $C$ is unique. For multivariate case, the copula density $C$ is obtained by

$$
c\left(F_{1},\left(x_{1}\right), \ldots, F_{n}\left(x_{n}\right)\right)=\frac{h\left(F_{1}^{(-1)}\left(u_{1}\right), \ldots, F_{n}^{(-1)}\left(u_{n}\right)\right)}{\prod_{i=1}^{n} f_{i}\left(F_{i}^{(-1)}\left(u_{i}\right)\right)} .
$$

where $C$ is the copula density, $h$ is the density function associated to $H$, and $f_{i}$ is the density function of each marginal distribution. In this study, we will focus only on the Gaussian copula function. In case of multivariate model, let $\Phi()$ be standard normal cumulative distribution, thus Gaussian copula density can be written as

$$
f_{(n)}(N)=\frac{1}{\left|R^{1 / 2}\right|} \exp \left\{\frac{-1}{2} \gamma \gamma^{\prime}\left(R^{-1}-I\right)\right\}\left\{\prod_{i=1}^{n} \exp \left(\frac{-1}{2} \gamma_{i}^{2}\right)\right\}^{-1}
$$

where $\gamma_{i}=\Phi^{-1}\left(F_{i}\left(x_{i}\right)\right)=\Phi^{-1}\left(u_{i}\right), u_{i}=F_{i}\left(x_{i}: \theta_{i}\right), R$ is correlation matrix, and $I$ is the identity matrix.

\subsection{Regime Switching Copula}

In dynamic financial time series exhibit different behaviors in different regimes, and lead to different dependencies over time; for this reason, the dependence structure of the variables may be determined by a hidden Markov chain with two regimes or more. Therefore, it is reasonable to extend the copula to Markov Switching (Hamilton [7]) and obtain Markov Switching copula. Thus, the model becomes more flexible since it allows the dependence copula parameter $\left(R_{c, t}^{S_{t}}\right)$ to be governed by an unobserved variable at time $t\left(S_{t}\right)$. Let $S_{t}$ be the state variable, and have two states $(k=2)$, namely high and low dependence regime. The joint distribution of $x_{1}, \ldots, X_{n}$ conditional on $S_{t}$, is defined as

$\left(x_{1}, \ldots, x_{n, t} \mid S_{t}=i\right) \sim c_{t}^{S_{t}}\left(u_{i t}, \ldots, u_{n T} \mid \theta_{c, t}^{S_{t}}, R_{c, t}^{S_{t}}\right), \quad i=1,2$

The unobservable regime $\left(s_{t}\right)$ is governed by the first order Markov chain, which is characterized by the following transition probabilities $(P)$

$$
P_{i j}=\operatorname{Pr}\left(S_{t+1}=j \mid S_{t+1}=i\right) \text { and } \sum_{j=1}^{2} P_{i j}=1 \text {, if } i, j=1,2
$$

where $p_{i j}$ is the probability of switching from regime $i$ to regime $j$, and these transition probabilities can be formed in a transition matrix $P$ , as follows:

$$
P=\left[\begin{array}{cc}
P_{11} & P_{12}=1-P_{11} \\
P_{21}=1-P_{22} & P_{22}
\end{array}\right]
$$

Following, Song (2000), the Gaussian copula density function from Eq.(6) can be rewritten in the likelihood function form as

$$
L_{(n)}\left(u_{1}, \ldots, u_{n} \mid \theta_{1}, \ldots, \theta_{n}, R\right)=\frac{1}{\left|R^{1 / 2}\right|} \prod_{i}^{T}\left(\exp \left\{-\frac{1}{2} \gamma^{\prime}\left(R^{-1}-I\right) \gamma\right\} \prod_{j=1}^{n} f_{i}\left(x_{i j} ; \theta_{j}\right)\right)
$$

where $f_{i}\left(x_{i j} ; \theta_{j}\right)$ is the density function obtained from the ARMA-GARCH step and we assume this function to be fix.

In this study, the method of Kim's filtering algorithm (Kim and Nelson [10]) is applied to filter the state variable $S_{t}$ and let $L_{(t)}\left(x_{1}, \ldots x_{n} \mid \theta_{1}, \ldots, \theta_{n}, R, v\right)$ and $L_{(n)}\left(x_{1}, \ldots x_{n} \mid \theta_{1}, \ldots, \theta_{n}, R\right)$ be $L(t)$ and $L(N)$ respectively, thus we can write the two regime Markov Switching copula log likelihood for Gaussian distribution as

$\log L_{N}\left(\theta_{n, S_{t}}, R_{n, S_{t}}, P\right)=\log L(N) \operatorname{Pr}\left(S_{t} \mid \theta_{n, t-1}, R_{n, t-1}, P\right)$

To evaluate the log-likelihood in Eq. (11), we need to calculate the weight $\operatorname{Pr}\left(S_{t} \mid \theta_{n, t-1}, R_{n, t-1}\right) \operatorname{Pr}\left(S_{t} \mid \theta_{t-1}, R_{t, t-1}, v_{t-1}\right) \quad$ for $S_{t}=1,2$ because the estimation of the Markov Switching copula needs inferences on the probabilities of $S_{t}$.

$$
\begin{aligned}
& \operatorname{Pr}\left(S_{t}=1 \mid \mathrm{w}_{t}\right)=\frac{\log L \mid \operatorname{Pr}\left(S_{t}=l \mid w_{t-1}\right)}{\sum_{k=1}^{2} \log L \mid \operatorname{Pr}\left(S_{t}=k \mid w_{t-1}\right)} \\
& \operatorname{Pr}\left(S_{t}=2 \mid w_{t}\right)=1-\operatorname{Pr}\left(S_{t}=1 \mid w_{t}\right)
\end{aligned}
$$

where $W$ is all the information set of the model. The Bayesian approach is applied in order to estimate transition matrix parameters and dependence parameters,

\subsection{Value at Risk, Expected Shortfall and Portfolio Optimization}

In order to analyze the risk, we calculate the empirical VaR and ES of an equally weighted portfolio with 8 assets. The VaR of portfolio can be written as

$\operatorname{VaR}_{\alpha}=\inf \{l \in R: P(L>l) \leq 1-\alpha\}$

where, $\alpha$ is a confidence level with a value [0,1] which presents the probability of Loss $L$ to exceed $l$ but not larger than $(1-\alpha)$. The minimize expected shortfalls with respect to maximize returns, which can be given by:

Min $E S=E\left[r \mid r \leq r_{\alpha}\right]$

$$
\begin{array}{r}
\text { Subject to } \quad r=w\left[r_{(1, t+1)}+\ldots+r_{(8, t+1)}\right] \\
w_{1}=w_{2}=w_{3}=w_{4}=\frac{1}{8}, 0 \leq w_{i} \leq 1, i=1, \ldots, 8
\end{array}
$$

where $r_{\alpha}$ is the lower $\alpha$ - quantile and $r_{i, t+1}$ is the return on individual asset at time $t+1$. Next, the optimal portfolios weights of the selected assets then are constructed under minimize expected shortfalls with respect to maximize returns, which can be given by:

$$
\begin{gathered}
\text { Min } E S=E\left[r \mid r \leq r_{\alpha}\right] \\
\text { Subject to } r=w_{1} r_{(1, t+1)}+\ldots+w_{8} r_{(8, t+1)} \\
w_{1}+w_{2}+w_{3}+w_{4}=1,0 \leq w_{i} \leq 1, i=1, \ldots, 8
\end{gathered}
$$

where $r_{\alpha}$ is the lower $\alpha$ - quantile and $r_{i, t+1}$ is the return on individual asset at time $t+1$.

\section{Data}

For the empirical analysis, daily data are eight price indexes obtained from the stock price index of five major countries in ASEAN namely, Bursa Malaysia KLCL (Malaysia), SET (Thailand), Ho Chi Minh (Vietnam), Straits Times (Singapore), and Jakarta composite (Indonesia) plus three countries, namely KOSPI (South Korea), NIKKEI 225 (Japan), and Shanghai SE Composite (China). The data period starts from Jan 1, 2007 - May 30, 2017. The returns of the daily closing price index are calculated by a 
continuous compound basis, defined as $r_{i, t}=\ln \left(P_{i, t} / P_{i, t-1}\right)$, where $P_{i, t}$ and $P_{i, t-1}$ are the closing price index of market $i=1, \ldots, 8$ for days $t$ and $t-1$, respectively. The plots of the returns in their respective markets are shown in Fig. 1, and clearly move in a similar manner.

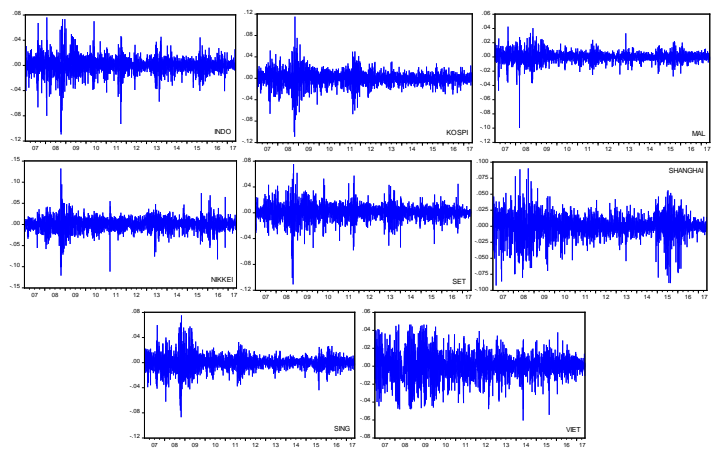

Figure 1 Stock price index returns

The descriptive statistics and unit root test for the stock price index return are reported in Table 1, respectively. The average returns of the stock price index are low and positive except for Vietnam is negative. The unit root test results based on Augmented Dickey-Fuller (ADF) test are reported in subsection of Table 1. Under the null hypothesis that daily stock index return has a unit root or nonstationary, all reject the null hypothesis at the $1 \%$ level of significance in every model specification.

Table 1 Descriptive statistics

\begin{tabular}{|c|c|c|c|c|c|c|c|c|}
\hline & INDO & KOSPI & MAL & $\underset{I}{\text { NIKKE }}$ & SET & $\begin{array}{c}\text { SHANGH } \\
\text { AI }\end{array}$ & SING & VIET \\
\hline Mean & $\begin{array}{c}0.0004 \\
2\end{array}$ & $\begin{array}{c}0.0001 \\
8\end{array}$ & $\begin{array}{c}1.75 \mathrm{E}- \\
04\end{array}$ & $\begin{array}{c}4.90 \mathrm{E}- \\
05\end{array}$ & $\begin{array}{c}3.08 \mathrm{E}- \\
04\end{array}$ & $5.54 \mathrm{E}-05$ & $\begin{array}{c}3.44 \mathrm{E}- \\
05\end{array}$ & $\begin{array}{c}-6.70 \mathrm{E}- \\
06\end{array}$ \\
\hline SD & 0.0134 & 0.0131 & 0.0075 & 0.0158 & 0.0122 & 0.0171 & 0.0114 & 0.0145 \\
\hline Max & 0.0763 & 0.1154 & 0.0426 & 0.1324 & 0.0755 & 0.0903 & 0.0753 & 0.0465 \\
\hline Min & 0.1095 & 0.1091 & -0.0996 & 0.1211 & 0.1109 & -0.0926 & 0.0869 & -0.0605 \\
\hline Skew & $\begin{array}{c}- \\
0.6462\end{array}$ & $\stackrel{-}{0.4418}$ & -1.1667 & $\begin{array}{c}- \\
0.5082\end{array}$ & $\begin{array}{c}- \\
0.6497\end{array}$ & -0.6343 & $\stackrel{-}{0.1768}$ & -0.1873 \\
\hline Kur & 11.399 & 11.988 & 18.623 & 11.250 & 11.078 & 7.4397 & 9.7175 & 4.4669 \\
\hline JB test & $\begin{array}{c}8,173 . \\
6 \\
\end{array}$ & $\begin{array}{c}9,232 . \\
4\end{array}$ & $\begin{array}{c}28,238 \\
.4\end{array}$ & $\begin{array}{c}7,819 . \\
5\end{array}$ & $\begin{array}{c}7,575 \\
9\end{array}$ & $2,412.7$ & $\begin{array}{c}5,120 \\
8\end{array}$ & 259.4 \\
\hline \multicolumn{9}{|l|}{$\begin{array}{l}\text { ADF- } \\
\text { Test: }\end{array}$} \\
\hline None & $\begin{array}{c}-.9 \\
46.94\end{array}$ & $\begin{array}{c}51.74 \\
9\end{array}$ & $\begin{array}{c}- \\
46.440\end{array}$ & $\begin{array}{c}- \\
53.973\end{array}$ & $\begin{array}{c}- \\
50.282\end{array}$ & -51.623 & $\begin{array}{c}- \\
50.503\end{array}$ & -40.834 \\
\hline $\begin{array}{l}\text { Trend \& } \\
\text { Interce } \\
\text { pt }\end{array}$ & $\begin{array}{c}- \\
46.96 \\
6\end{array}$ & $\underset{0}{51.74}$ & $\stackrel{-}{46.451}$ & $\begin{array}{c}- \\
54.000\end{array}$ & $\begin{array}{c}- \\
50.294\end{array}$ & -51.605 & $\begin{array}{c}- \\
50.486\end{array}$ & -40.844 \\
\hline $\begin{array}{l}\text { Interce } \\
\text { pt }\end{array}$ & $\begin{array}{c}- \\
46.97 \\
3\end{array}$ & $\begin{array}{c}51.74 \\
9\end{array}$ & $\begin{array}{c}-\cdot \\
46.455\end{array}$ & $\begin{array}{c}-\overline{-} \\
53.963\end{array}$ & $\begin{array}{c}-\overline{304} \\
50.30\end{array}$ & -51.614 & $\begin{array}{c}-\overline{495} \\
50.4\end{array}$ & -40.826 \\
\hline
\end{tabular}

Note: Entries in bold are significant at the $1 \%$ level.

\section{Empirical results}

This section presents the Markov Switching Multivariate Copula model for eight stock price index returns. Table 3 illustrates the estimated coefficients for the $\operatorname{ARMA}(p, q)$-EGARCH $(1,1)$ with student $t$ distribution for each stock price index returns series. The optimum lag length for $\operatorname{ARMA}(p, q)$-EGARCH $(1,1)$ is selected by the minimum value of Akaike information criteria (AIC) and Bayesian information criterion (BIC) information, and found that the returns on SET and SING are ARMA(1,1)EGARCH(1,1), MAL and NIKKEI is ARMA(1,0)-GARCH(1,1), while SHANGHAI, INDO, KOSPI and VIET satisfied

\section{Table 2 Estimate ARMA(p,q)-EGARCH $(1,1)$ with $t$ Distribution}

\begin{tabular}{|c|c|c|c|c|c|c|c|c|}
\hline & SET & MAL & VIET & INDO & SING & KOSPI & NIKKEI & $\begin{array}{c}\text { SHANGH } \\
\text { AI }\end{array}$ \\
\hline \multirow[t]{2}{*}{$\mu$} & 0.0005 & 0.0003 & 0.0000 & 0.0001 & 0.0001 & 0.0002 & 0.0005 & 0.0005 \\
\hline & $1.465^{*}$ & $\begin{array}{l}3.448^{*} \\
* *\end{array}$ & $1.399^{*}$ & $0.288^{*}$ & $0.32^{*}$ & $2.071^{*}$ & $2.24^{*}$ & $2.328^{*}$ \\
\hline \multirow[t]{2}{*}{$\operatorname{AR}(1)$} & 0.4150 & 0.0956 & 1.0000 & & 0.1098 & 0.6540 & -0.0409 & \\
\hline & $1.082^{*}$ & $\begin{array}{c}4.96 * * \\
*\end{array}$ & $\begin{array}{c}8.934^{* *} \\
*\end{array}$ & & $0.826^{*}$ & $\underset{* *}{4.815^{*}}$ & $2.129^{*}$ & \\
\hline $\operatorname{AR}(2)$ & & & $\begin{array}{r}-0.0543 \\
0.498^{*}\end{array}$ & & & $\begin{array}{l}0.0010 \\
0.055^{*}\end{array}$ & & \\
\hline
\end{tabular}

\begin{tabular}{|c|c|c|c|c|c|c|c|c|}
\hline \multirow[t]{2}{*}{ MA(1) } & \multicolumn{2}{|l|}{-0.3932} & -0.8210 & 0.1814 & 0.0730 & \multicolumn{3}{|l|}{0.6748} \\
\hline & \multicolumn{2}{|l|}{-1.0130} & $7.169 * *$ & $\begin{array}{l}8.698^{* *} \\
*\end{array}$ & $0.546^{*}$ & $\underset{* *}{4.983^{*}}$ & & \\
\hline \multirow[t]{2}{*}{$\mathrm{MA}(2)$} & & & -0.0977 & 0.0135 & & & & \\
\hline & & & $0.895^{*}$ & $0.681^{*}$ & & & & \\
\hline \multirow[t]{2}{*}{$\omega$} & 0.0000 & 0.0000 & 0.0000 & 0.0000 & 0.0000 & 0.0000 & 0.0000 & 0.0000 \\
\hline & $3.012^{*}$ & $\underset{* *}{3.552^{*}}$ & $\begin{array}{c}3.675^{* *} \\
*\end{array}$ & $\begin{array}{c}3.651^{* *} \\
*\end{array}$ & ${ }^{3.65 *}$ & $2.382^{*}$ & $\begin{array}{c}3.768^{* * *} \\
*\end{array}$ & $2.151^{*}$ \\
\hline \multirow[t]{2}{*}{$\alpha$} & 0.1102 & 0.1086 & 0.1801 & 0.1754 & 0.1779 & 0.0606 & 0.1069 & 0.0571 \\
\hline & $\begin{array}{c}7.833^{* *} \\
*\end{array}$ & $\underset{* *}{6.523^{*}}$ & $\begin{array}{c}7.961^{* *} \\
*\end{array}$ & $7.97 * * *$ & $\underset{* *}{7.956^{*}}$ & $5.862^{*}$ & $\begin{array}{c}6.929 * * \\
*\end{array}$ & $5.757^{* * *}$ \\
\hline \multirow[t]{2}{*}{$\beta$} & 0.8876 & 0.8786 & 0.8059 & 0.8106 & 0.8082 & 0.9370 & 0.8719 & 0.9460 \\
\hline & $\underset{* *}{69.651^{*}}$ & $51.37^{*}$ & $\underset{* *}{34.238^{*}}$ & $\underset{* *}{35.317^{*}}$ & $\underset{* *}{34.67 *}$ & $\underset{*}{91.772}$ & $\underset{* *}{50.811^{*}}$ & $\underset{* *}{116.277^{*}}$ \\
\hline \multirow[t]{2}{*}{$\gamma$} & 5.3370 & 5.0140 & $\begin{array}{c}10.000 \\
0\end{array}$ & $\begin{array}{c}10.000 \\
0\end{array}$ & $\begin{array}{c}10.000 \\
0\end{array}$ & 5.0750 & 5.5280 & 3.8480 \\
\hline & $\begin{array}{c}8.973^{* *} \\
*\end{array}$ & $\begin{array}{l}9.785^{*} \\
* *\end{array}$ & $\begin{array}{c}6.091^{* *} \\
*\end{array}$ & $\underset{*}{6.126^{* *}}$ & $\underset{* *}{6.228^{*}}$ & $8.717^{*}$ & $\underset{*}{8.421^{* *}}$ & $11.35{ }^{* * *}$ \\
\hline \multirow{2}{*}{$\begin{array}{c}\log L \\
\text { normaliz } \\
\text { ed }\end{array}$} & $8,673.3$ & $\begin{array}{c}10,038 \\
.7\end{array}$ & $8,158.2$ & $8,146.4$ & $\begin{array}{c}8,146 . \\
5\end{array}$ & $\begin{array}{c}8,549 . \\
0\end{array}$ & $7,904.3$ & $7,787.5$ \\
\hline & 3.193 & 3.696 & 3.004 & 2.999 & 2.999 & 3.148 & 2.910 & 2.867 \\
\hline
\end{tabular}

Note: $* * *, * *, *$ denote significant at the $1 \%, 5 \%$ and $10 \%$, respectively.

Table 3 Empirical copula parameters

\begin{tabular}{|c|c|c|c|c|c|c|c|c|}
\hline $\begin{array}{c}\text { Regime } \\
1\end{array}$ & SET & MAL & VIET & INDO & SING & KOSPI & $\begin{array}{c}\text { NIKKE } \\
\text { I }\end{array}$ & $\begin{array}{c}\text { SHANGH } \\
\text { AI } \\
\end{array}$ \\
\hline \multirow[t]{2}{*}{ SET } & 1.0000 & 0.3900 & 0.1057 & 0.1056 & 0.1001 & 0.3820 & 0.3323 & 0.2128 \\
\hline & & $\begin{array}{c}(17.32 \\
2)\end{array}$ & (3.291) & $\begin{array}{c}(3.287 \\
)\end{array}$ & $\begin{array}{c}(5.198 \\
)\end{array}$ & $\begin{array}{c}(24.78 \\
4)\end{array}$ & $\begin{array}{c}(20.53 \\
7)\end{array}$ & $(9.498)$ \\
\hline \multirow[t]{2}{*}{ MAL } & 0.3900 & 1.0000 & 0.1296 & 0.1352 & 0.1290 & 0.4548 & 0.4122 & 0.2338 \\
\hline & $\begin{array}{c}(17.32 \\
2)\end{array}$ & & (3.984) & $\begin{array}{c}(4.880 \\
)\end{array}$ & $\begin{array}{c}(6.695 \\
)\end{array}$ & $\begin{array}{c}(21.49 \\
5)\end{array}$ & $\begin{array}{c}(17.82 \\
2)\end{array}$ & $(7.258)$ \\
\hline \multirow[t]{2}{*}{ VIET } & 0.1057 & 0.1296 & 1.0000 & 0.9900 & 0.9900 & 0.1410 & 0.1820 & 0.0889 \\
\hline & (3.291) & (3.984) & & $\mathrm{NaN}$ & $\mathrm{NaN}$ & $\begin{array}{c}(4.944 \\
)\end{array}$ & $\begin{array}{c}(5.593 \\
)\end{array}$ & $(2.740)$ \\
\hline \multirow[t]{2}{*}{ Indo } & 0.1056 & 0.1352 & 0.9900 & 1.0000 & 0.9900 & $\begin{array}{c}0.140 \\
7\end{array}$ & 0.1818 & 0.0842 \\
\hline & (3.287) & $(4.880)$ & $\mathrm{NaN}$ & & $\mathrm{NaN}$ & 4.903) & $\begin{array}{c}(5.536 \\
)\end{array}$ & $(2.356)$ \\
\hline \multirow[t]{2}{*}{ SING } & 0.1001 & 0.1290 & 0.9900 & 0.9900 & 1.0000 & 0.1318 & 0.1814 & 0.0910 \\
\hline & (5.198) & (6.695) & $\mathrm{NaN}$ & $\mathrm{NaN}$ & & $\begin{array}{c}(6.937 \\
)\end{array}$ & $\stackrel{(9.735}{)}$ & $(4.683)$ \\
\hline \multirow[t]{2}{*}{ KOSPI } & 0.3820 & 0.4548 & 0.1410 & 0.1407 & 0.1318 & 1.0000 & 0.5718 & 0.3131 \\
\hline & $\begin{array}{c}(24.78 \\
4)\end{array}$ & $\begin{array}{c}(21.49 \\
5)\end{array}$ & $(4.944)$ & $\begin{array}{c}(4.903 \\
)\end{array}$ & $\begin{array}{c}(6.937 \\
)\end{array}$ & & $\begin{array}{c}(49.71 \\
6)\end{array}$ & $(15.050)$ \\
\hline \multirow[t]{2}{*}{ NIKKEI } & 0.3323 & 0.4122 & 0.1820 & 0.1818 & 0.1814 & 0.5718 & 1.0000 & 0.2728 \\
\hline & $\begin{array}{c}(20.53 \\
7)\end{array}$ & $\begin{array}{c}(17.82 \\
2)\end{array}$ & (5.593) & $\begin{array}{c}(5.536 \\
)\end{array}$ & $\begin{array}{c}9.735 \\
)\end{array}$ & $\begin{array}{c}(49.71 \\
6)\end{array}$ & & (12.309) \\
\hline \multirow[t]{2}{*}{$\begin{array}{c}\text { SHANGH } \\
\text { AI }\end{array}$} & 0.2128 & 0.2338 & 0.0889 & 0.0842 & 0.0910 & 0.3131 & 0.2728 & 1.0000 \\
\hline & (9.498) & $(7.258)$ & $(2.740)$ & $\begin{array}{c}(2.356 \\
)\end{array}$ & $\begin{array}{c}(4.683 \\
)\end{array}$ & $\begin{array}{c}(15.05 \\
0) \\
\end{array}$ & $\begin{array}{c}(12.30 \\
9)\end{array}$ & \\
\hline
\end{tabular}

\begin{tabular}{|c|c|c|c|c|c|c|c|c|}
\hline$\underset{2}{\text { Regime }}$ & SET & MAL & VIET & INDO & SING & KOSPI & $\underset{\text { I }}{\text { NIKKE }}$ & $\begin{array}{c}\text { SHANGH } \\
\text { AI }\end{array}$ \\
\hline \multirow[t]{2}{*}{ SET } & 1.0000 & 0.0771 & -0.1323 & 0.1201 & 0.2818 & 0.2503 & 0.0858 & -0.1338 \\
\hline & & $\begin{array}{c}(- \\
0.437)\end{array}$ & $\begin{array}{c}(- \\
0.658)\end{array}$ & $\begin{array}{c}(- \\
0.533)\end{array}$ & $\begin{array}{c}(- \\
1.842)\end{array}$ & $\begin{array}{c}0.263 \\
)\end{array}$ & $\stackrel{(0.057}{)}$ & $(-0.911)$ \\
\hline \multirow[t]{2}{*}{ MAL } & 0.0771 & 1.0000 & 0.0561 & 0.0592 & 0.2703 & 0.3664 & 0.1545 & -0.0688 \\
\hline & $\begin{array}{c}(- \\
0.437)\end{array}$ & & $(0.391)$ & $\begin{array}{c}0.412 \\
)\end{array}$ & $\begin{array}{c}(2.030 \\
)\end{array}$ & $\begin{array}{c}(1.530 \\
)\end{array}$ & $\begin{array}{c}0.475 \\
)\end{array}$ & $(-0.487)$ \\
\hline \multirow[t]{2}{*}{ VIET } & 0.1323 & 0.0561 & 1.0000 & 0.9900 & 0.8262 & 0.0698 & $\stackrel{(0.020}{9}$ & 0.0964 \\
\hline & $\begin{array}{c}(- \\
0.658)\end{array}$ & $(0.391)$ & & $\mathrm{NaN}$ & $\begin{array}{c}(16.22 \\
0)\end{array}$ & $\begin{array}{c}0.497 \\
)\end{array}$ & $\stackrel{(0.191}{)}$ & $(0.0964$ \\
\hline \multirow[t]{2}{*}{ Indo } & 0.1201 & 0.0592 & 0.9900 & 1.0000 & 0.8246 & 0.1396 & 0.1235 & $(0.716)$ \\
\hline & $\begin{array}{c}(- \\
0.533)\end{array}$ & $(0.412)$ & $\mathrm{NaN}$ & & $\begin{array}{c}(14.48 \\
8)\end{array}$ & $\stackrel{(1.036}{)}$ & $\stackrel{(1.156}{)}$ & $(0.707)$ \\
\hline \multirow[t]{2}{*}{ SING } & $0 . \overline{2818}$ & 0.2703 & 0.8262 & 0.8246 & 1.0000 & 0.0874 & 0.0372 & 0.1273 \\
\hline & $\begin{array}{c}(- \\
1.842)\end{array}$ & (2.030) & $\begin{array}{c}(16.22 \\
0)\end{array}$ & $\begin{array}{c}(14.48 \\
8)\end{array}$ & & $\begin{array}{c}0.358 \\
)\end{array}$ & $\begin{array}{c}(0.117 \\
)\end{array}$ & $(0.958)$ \\
\hline \multirow[t]{2}{*}{ KOSPI } & 0.2503 & 0.3664 & 0.0698 & 0.1396 & 0.0874 & 1.0000 & 0.6774 & -0.1644 \\
\hline & $(0.263)$ & (1.530) & $(0.497)$ & $\stackrel{(1.036}{)}$ & $\begin{array}{c}(0.358 \\
)\end{array}$ & & $\begin{array}{c}(6.988 \\
)\end{array}$ & $(-0.930)$ \\
\hline \multirow[t]{2}{*}{ NIKKEI } & 0.0858 & 0.1545 & $\begin{array}{c}0.020 \\
9\end{array}$ & 0.1235 & 0.0372 & 0.6774 & 1.0000 & 0.1748 \\
\hline & $(0.057)$ & $(0.475)$ & $(0.191)$ & $\underset{f}{(1.156}$ & $\begin{array}{c}0.117 \\
)\end{array}$ & $\begin{array}{c}(6.988 \\
)\end{array}$ & & $(0.841)$ \\
\hline \multirow[t]{3}{*}{$\begin{array}{c}\text { SHANGH } \\
\text { AI }\end{array}$} & 0.1338 & 0.0688 & $\begin{array}{c}(0.096 \\
4\end{array}$ & 0.0952 & 0.1273 & $\hat{0.1644}$ & 0.1748 & 1.0000 \\
\hline & $\begin{array}{c}(- \\
0.911)\end{array}$ & $\begin{array}{c}(- \\
0.487)\end{array}$ & & $\begin{array}{c}0.707 \\
)\end{array}$ & $\begin{array}{c}0.958 \\
)\end{array}$ & $\begin{array}{c}(- \\
0.930)\end{array}$ & $\begin{array}{c}(0.841 \\
)\end{array}$ & \\
\hline & $\begin{array}{c}\text { Regim } \\
\text { e1 }\end{array}$ & $\begin{array}{c}\text { Regim } \\
\text { e2 }\end{array}$ & $\begin{array}{c}\begin{array}{c}\text { Durati } \\
\text { on }\end{array} \\
\end{array}$ & & & & & \\
\hline$\underset{1}{\text { Regime }}$ & $\begin{array}{c}0.9999 \\
(38.14 \\
6)\end{array}$ & 0.0001 & 38.15 & & & & & \\
\hline$\underset{2}{\text { Regime }}$ & 0.0321 & $\begin{array}{c}0.9679 \\
(23.12 \\
4)\end{array}$ & 23.12 & & & & & \\
\hline
\end{tabular}

Source: Calculation

$\operatorname{ARMA}(0,0), \operatorname{ARMA}(0,2), \operatorname{ARMA}(2,1)$, and $\operatorname{ARMA}(2,2)$ with $\operatorname{EGARCH}(1,1)$ respectively. Since the asymmetry exists but indicates the leverage. The best-fit of $\operatorname{ARMA}(p, q)$-EGARCH(1,1) will give the standardized residuals to be transformed into a uniform distribution in $(0,1)$. 
Table 3 shows the empirical copula parameters of multivariate Gaussian copula parameters with regime switching. The empirical results show that the value of the matrix dependence parameter in regime 1 is higher than regime 2 . Therefore, the regime 1 should be the high dependence regime, while regime 2 should be the low dependence regime.

From the Markov switching model, the estimated transition probability matrix of these stock price index return are also reported at the end of Table 3. The $\operatorname{Pr}\left(S_{t}=1 \mid S_{t}=1\right)$ is $99.99 \%$ and $\operatorname{Pr}\left(S_{t}=2 \mid S_{t}=2\right)$ is $96.79 \%$, meaning the probabilities of regime switching between these two regimes are less than $5 \%$. The results indicate that both regimes are persistent because of the high values obtained from the probabilities. Consequently, the duration of staying in regime 1 and 2 is 38.15 and 23.12 days, respectively.

Table 4 Optimal Portfolio's weight

\begin{tabular}{|c|c|c|c|c|c|c|c|c|c|c|}
\hline $\begin{array}{c}\begin{array}{c}\text { Regim } \\
\text { e1 }\end{array} \\
\begin{array}{c}\text { portfo } \\
\text { lio }\end{array} \\
\end{array}$ & SET & MAL & $\begin{array}{c}\mathrm{VIE} \\
\mathrm{T}\end{array}$ & $\begin{array}{c}\text { IND } \\
0\end{array}$ & $\underset{G}{\text { SIN }}$ & $\begin{array}{c}\text { KOS } \\
\text { PI }\end{array}$ & $\underset{\text { EI }}{\text { NIKK }}$ & $\begin{array}{c}\text { SHANG } \\
\text { HAI }\end{array}$ & $\begin{array}{c}\text { Retu } \\
\text { rn }\end{array}$ & Risk \\
\hline 1 & $\begin{array}{c}0.16 \\
36\end{array}$ & $\begin{array}{c}0.10 \\
28\end{array}$ & $\begin{array}{c}0.05 \\
19\end{array}$ & $\begin{array}{c}0.21 \\
26\end{array}$ & $\begin{array}{c}0.00 \\
00\end{array}$ & $\begin{array}{c}0.09 \\
20\end{array}$ & $\begin{array}{c}0.11 \\
05\end{array}$ & 0.2667 & $\begin{array}{c}0.00 \\
02\end{array}$ & $\begin{array}{c}0.14 \\
07\end{array}$ \\
\hline 2 & $\begin{array}{c}0.13 \\
24\end{array}$ & $\begin{array}{c}0.09 \\
42\end{array}$ & $\begin{array}{c}0.14 \\
39\end{array}$ & $\begin{array}{c}0.11 \\
27\end{array}$ & $\begin{array}{c}0.00 \\
00\end{array}$ & $\begin{array}{c}0.06 \\
21\end{array}$ & $\begin{array}{c}0.18 \\
88\end{array}$ & 0.2660 & $\begin{array}{c}0.00 \\
00\end{array}$ & $\begin{array}{c}0.14 \\
16\end{array}$ \\
\hline 3 & $\begin{array}{c}0.11 \\
19\end{array}$ & $\begin{array}{c}0.08 \\
45\end{array}$ & $\begin{array}{c}0.14 \\
61\end{array}$ & $\begin{array}{c}0.09 \\
41\end{array}$ & $\begin{array}{c}0.00 \\
00\end{array}$ & $\begin{array}{c}0.02 \\
27\end{array}$ & $\begin{array}{c}0.28 \\
13\end{array}$ & 0.2593 & $\begin{array}{c}0.00 \\
01\end{array}$ & $\begin{array}{c}0.14 \\
40\end{array}$ \\
\hline 4 & $\begin{array}{c}0.07 \\
77\end{array}$ & $\begin{array}{c}0.06 \\
48\end{array}$ & $\begin{array}{c}0.23 \\
17\end{array}$ & $\begin{array}{c}0.00 \\
00\end{array}$ & $\begin{array}{c}0.00 \\
00\end{array}$ & $\begin{array}{c}0.00 \\
00\end{array}$ & $\begin{array}{c}0.35 \\
26\end{array}$ & 0.2732 & $\begin{array}{c}0.00 \\
03\end{array}$ & $\begin{array}{c}0.14 \\
77\end{array}$ \\
\hline 5 & $\begin{array}{c}0.03 \\
35\end{array}$ & $\begin{array}{c}0.03 \\
86\end{array}$ & $\begin{array}{c}0.22 \\
33\end{array}$ & $\begin{array}{c}0.00 \\
00\end{array}$ & $\begin{array}{c}0.00 \\
00\end{array}$ & $\begin{array}{c}0.00 \\
00\end{array}$ & $\begin{array}{c}0.43 \\
39\end{array}$ & 0.2706 & $\begin{array}{c}0.00 \\
04\end{array}$ & $\begin{array}{c}0.15 \\
25\end{array}$ \\
\hline 6 & $\begin{array}{c}0.00 \\
00\end{array}$ & $\begin{array}{c}0.01 \\
24\end{array}$ & $\begin{array}{c}0.20 \\
45\end{array}$ & $\begin{array}{c}0.00 \\
00\end{array}$ & $\begin{array}{c}0.00 \\
00\end{array}$ & $\begin{array}{c}0.00 \\
00\end{array}$ & $\begin{array}{c}0.52 \\
06\end{array}$ & 0.2625 & $\begin{array}{c}0.00 \\
06\end{array}$ & $\begin{array}{c}0.15 \\
84\end{array}$ \\
\hline 7 & $\begin{array}{c}0.00 \\
00\end{array}$ & $\begin{array}{c}0.00 \\
00\end{array}$ & $\begin{array}{c}0.14 \\
63\end{array}$ & $\begin{array}{c}0.00 \\
00\end{array}$ & $\begin{array}{c}0.00 \\
00\end{array}$ & $\begin{array}{c}0.00 \\
00\end{array}$ & $\begin{array}{c}0.63 \\
13\end{array}$ & 0.2224 & $\begin{array}{c}0.00 \\
07\end{array}$ & $\begin{array}{c}0.16 \\
67\end{array}$ \\
\hline 8 & $\begin{array}{c}0.00 \\
00\end{array}$ & $\begin{array}{c}0.00 \\
00\end{array}$ & $\begin{array}{c}0.08 \\
13\end{array}$ & $\begin{array}{c}0.00 \\
00\end{array}$ & $\begin{array}{c}0.00 \\
00\end{array}$ & $\begin{array}{c}0.00 \\
00\end{array}$ & $\begin{array}{c}0.74 \\
83\end{array}$ & 0.1704 & $\begin{array}{c}0.00 \\
08\end{array}$ & $\begin{array}{c}0.17 \\
77\end{array}$ \\
\hline 9 & $\begin{array}{c}0.00 \\
00\end{array}$ & $\begin{array}{c}0.00 \\
00\end{array}$ & $\begin{array}{c}0.03 \\
26\end{array}$ & $\begin{array}{c}0.00 \\
00\end{array}$ & $\begin{array}{c}0.00 \\
00\end{array}$ & $\begin{array}{c}0.00 \\
00\end{array}$ & $\begin{array}{c}0.87 \\
13\end{array}$ & 0.0961 & $\begin{array}{c}0.00 \\
10\end{array}$ & $\begin{array}{c}0.19 \\
14\end{array}$ \\
\hline 10 & $\begin{array}{c}0.00 \\
00\end{array}$ & $\begin{array}{c}0.00 \\
00\end{array}$ & $\begin{array}{c}0.00 \\
00\end{array}$ & $\begin{array}{c}0.00 \\
00\end{array}$ & $\begin{array}{c}0.00 \\
00\end{array}$ & $\begin{array}{c}0.00 \\
00\end{array}$ & $\begin{array}{c}1.00 \\
00\end{array}$ & 0.0000 & $\begin{array}{c}0.00 \\
11\end{array}$ & $\begin{array}{c}0.20 \\
84\end{array}$ \\
\hline
\end{tabular}

\begin{tabular}{|c|c|c|c|c|c|c|c|c|c|c|}
\hline $\begin{array}{c}\begin{array}{c}\text { Regim } \\
\text { e2 }\end{array} \\
\text { portfo } \\
\text { lio }\end{array}$ & SET & MAL & $\begin{array}{c}\mathrm{VIE} \\
\mathrm{T}\end{array}$ & $\begin{array}{c}\text { IND } \\
0\end{array}$ & $\begin{array}{c}\text { SIN } \\
\text { G }\end{array}$ & $\begin{array}{c}\text { KOS } \\
\text { PI }\end{array}$ & $\begin{array}{c}\text { NIKK } \\
\text { EI }\end{array}$ & $\begin{array}{c}\text { SHANG } \\
\text { HAI }\end{array}$ & $\begin{array}{c}\text { Retu } \\
\text { rn }\end{array}$ & Risk \\
\hline 1 & $\begin{array}{c}0.26 \\
67\end{array}$ & $\begin{array}{c}0.18 \\
53\end{array}$ & $\begin{array}{c}0.08 \\
60\end{array}$ & $\begin{array}{c}0.00 \\
00\end{array}$ & $\begin{array}{c}0.11 \\
04\end{array}$ & $\begin{array}{c}0.03 \\
51\end{array}$ & $\begin{array}{c}0.10 \\
70\end{array}$ & 0.2095 & $\begin{array}{c}0.00 \\
02\end{array}$ & $\begin{array}{c}0.10 \\
91\end{array}$ \\
\hline 2 & $\begin{array}{c}0.25 \\
33\end{array}$ & $\begin{array}{c}0.18 \\
04\end{array}$ & $\begin{array}{c}0.12 \\
08\end{array}$ & $\begin{array}{c}0.00 \\
00\end{array}$ & $\begin{array}{c}0.15 \\
43\end{array}$ & $\begin{array}{c}0.01 \\
86\end{array}$ & $\begin{array}{c}0.11 \\
44\end{array}$ & 0.1581 & $\begin{array}{c}0.00 \\
00\end{array}$ & $\begin{array}{c}0.11 \\
04\end{array}$ \\
\hline 3 & $\begin{array}{c}0.24 \\
13\end{array}$ & $\begin{array}{c}0.14 \\
18\end{array}$ & $\begin{array}{c}0.15 \\
44\end{array}$ & $\begin{array}{c}0.00 \\
00\end{array}$ & $\begin{array}{c}0.21 \\
41\end{array}$ & $\begin{array}{c}0.00 \\
00\end{array}$ & $\begin{array}{c}0.12 \\
47\end{array}$ & 0.1237 & $\begin{array}{c}0.00 \\
02\end{array}$ & $\begin{array}{c}0.11 \\
37\end{array}$ \\
\hline 4 & $\begin{array}{c}0.23 \\
98\end{array}$ & $\begin{array}{c}0.12 \\
13\end{array}$ & $\begin{array}{c}0.21 \\
57\end{array}$ & $\begin{array}{c}0.00 \\
00\end{array}$ & $\begin{array}{c}0.23 \\
10\end{array}$ & $\begin{array}{c}0.00 \\
00\end{array}$ & $\begin{array}{c}0.11 \\
82\end{array}$ & 0.0740 & $\begin{array}{c}0.00 \\
04\end{array}$ & $\begin{array}{c}0.11 \\
93\end{array}$ \\
\hline 5 & $\begin{array}{c}0.21 \\
15\end{array}$ & $\begin{array}{c}0.12 \\
07\end{array}$ & $\begin{array}{c}0.28 \\
76\end{array}$ & $\begin{array}{c}0.00 \\
00\end{array}$ & $\begin{array}{c}0.24 \\
39\end{array}$ & $\begin{array}{c}0.00 \\
00\end{array}$ & $\begin{array}{c}0.09 \\
08\end{array}$ & 0.0455 & $\begin{array}{c}0.00 \\
06\end{array}$ & $\begin{array}{c}0.12 \\
69\end{array}$ \\
\hline 6 & $\begin{array}{c}0.18 \\
89\end{array}$ & $\begin{array}{c}0.10 \\
86\end{array}$ & $\begin{array}{c}0.32 \\
90\end{array}$ & $\begin{array}{c}0.00 \\
00\end{array}$ & $\begin{array}{c}0.28 \\
58\end{array}$ & $\begin{array}{c}0.00 \\
00\end{array}$ & $\begin{array}{c}0.08 \\
14\end{array}$ & 0.0062 & $\begin{array}{c}0.00 \\
07\end{array}$ & $\begin{array}{c}0.13 \\
71\end{array}$ \\
\hline 7 & $\begin{array}{c}0.15 \\
81\end{array}$ & $\begin{array}{c}0.07 \\
65\end{array}$ & $\begin{array}{c}0.46 \\
61\end{array}$ & $\begin{array}{c}0.00 \\
00\end{array}$ & $\begin{array}{c}0.24 \\
55\end{array}$ & $\begin{array}{c}0.00 \\
00\end{array}$ & $\begin{array}{c}0.05 \\
38\end{array}$ & 0.0000 & $\begin{array}{c}0.00 \\
09\end{array}$ & $\begin{array}{c}0.14 \\
99\end{array}$ \\
\hline 8 & $\begin{array}{c}0.10 \\
45\end{array}$ & $\begin{array}{c}0.05 \\
95\end{array}$ & $\begin{array}{c}0.54 \\
73\end{array}$ & $\begin{array}{c}0.00 \\
00\end{array}$ & $\begin{array}{c}0.26 \\
56\end{array}$ & $\begin{array}{c}0.00 \\
00\end{array}$ & $\begin{array}{c}0.02 \\
31\end{array}$ & 0.0000 & $\begin{array}{c}0.00 \\
11\end{array}$ & $\begin{array}{c}0.16 \\
61\end{array}$ \\
\hline 9 & $\begin{array}{c}0.07 \\
05\end{array}$ & $\begin{array}{c}0.00 \\
84\end{array}$ & $\begin{array}{c}0.61 \\
03\end{array}$ & $\begin{array}{c}0.00 \\
00\end{array}$ & $\begin{array}{c}0.31 \\
08\end{array}$ & $\begin{array}{c}0.00 \\
00\end{array}$ & $\begin{array}{c}0.00 \\
00\end{array}$ & 0.0000 & $\begin{array}{c}0.00 \\
13\end{array}$ & $\begin{array}{c}0.18 \\
57\end{array}$ \\
\hline 10 & $\begin{array}{c}0.00 \\
00\end{array}$ & $\begin{array}{c}0.00 \\
00\end{array}$ & $\begin{array}{c}1.00 \\
00\end{array}$ & $\begin{array}{c}0.00 \\
00\end{array}$ & $\begin{array}{c}0.00 \\
00\end{array}$ & $\begin{array}{c}0.00 \\
00\end{array}$ & $\begin{array}{c}0.00 \\
00\end{array}$ & 0.0000 & $\begin{array}{c}0.00 \\
15\end{array}$ & $\begin{array}{c}0.21 \\
11\end{array}$ \\
\hline
\end{tabular}

Source: Calculation

Table 4 shows the optimal portfolio's weight for 2 regimes. Figure 2 displays the efficiency frontier for two regimes from the 10 portfolios in the Table 5. The empirical results suggest that in regime 1, the risk lover investors should allocate the investment in NIKKEI 87.13\%, SHANGHAI 9.61\% and VIET 3.26\%, but the risk-averse investor should allocate money in SHANGHAI 26.6, NIKKEI 18.8, VIET 14.4, SET 13.2, INDO 11.3, MAL 9.42, and KOSPI 6.21\%, respectively. However, in regime 2 the optimum portfolios differ from regime 1. In market upturn period, the risk lover investors should allocate the investment in VIET 61.03\%, SING 31.08\%, SET $7.05 \%$, and MAL0.84\%, while but the risk-averse investor should allocate money in SET 25.3\%, MAL18.04\%, SHANGHAI 15.81\%, SING $15.43 \%$, VIET $12.08 \%$, NIKKEI $11.44 \%$, KOSPI $1.86 \%$, respectively.

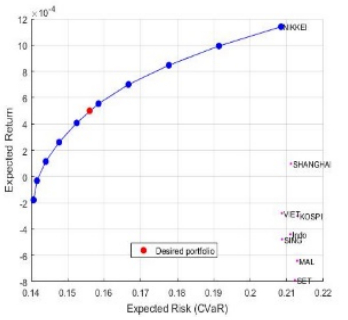

(a) Regime 1

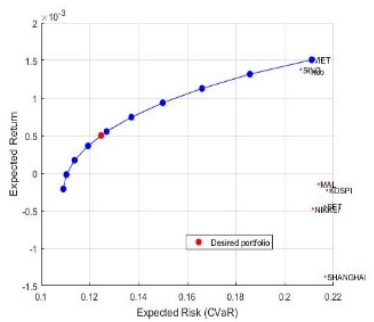

(b) Regime 2

Figure 2. Efficient frontier for two regimes

\section{Conclusions}

In this paper, we investigate the optimum portfolio of ASEAN+3 index return consisting of Bursa Malaysia KLCI (Malaysia), SET (Thailand), Ho Chi Minh (Vietnam), Straits Times (Singapore), Jakarta composite (Indonesia), KOSPI (South Korea), NIKKEI 225 (Japan), and Shanghai SE Composite (China). In this study was applied ARMA-EGARCH with $t$ distribution model with Markov Switching with high dimension Gaussian copula in order to measure a dependency of eight price index returns, and then construct the optimum portfolio for the market downturn and upturn regime. The results show that, in market downturn situation the risk lover investor should invest high proportion in NIKKEI 225, but risk averse investor should diversify money in many market with different proportion except for Straits Times of Singapore. In case of market upturn situation, the risk lover investor should invest high proportion in Ho Chi Minh and Straits Times of Singapore but risk averse investor should diversify money in many market with different proportion except for Jakarta composite of Indonesia. According to drawbacks EGARCH of Nelson [8,9] such as incorrect conditions, no clear explanation which lead to misleading interpretation (McAleer and Hafner [10], Chang and McAleer [11]). The future research should apply the recent development model of EGARCH of McAleer and Hafner [10] derived from a random coefficient complex nonlinear moving average process instead of the traditional EGARCH, and compare the empirical results.

\section{Acknowledgement}

We are grateful for financial support from Puay Ungpakorn Centre of Excellence in Econometrics, Faculty of Economics, Chiang Mai University.

\section{References}

[1] [1] R. Click and M.G. Plummer, "Stock market integration in ASEAN after the financial crisis," Journal of Asian Economics, 16(1), pp. 5-28, February 2005.

[2] [2] B.A. Karim and Z.A. Karim, "Integration of ASEAN-5 stock markets: a revisit," Asian Academy of Management Journal of Accounting and Finance, 8(2), pp. 21-41, 2012.

[3] [3] The World Bank, "Market capitalization of listed domestic companies (current US\$) http://data.worldbank.org/indicator/CM.MKT.LCAP. CD, (2016)

[4] [4] L. Deng, C. Ma, and W. Yang. Portfolio Optimization via Pair Copula-GARCH-EVT-CVaR Model. Systems Engineering Procedia. Vol.2 (2011), p. 171 - 181.

[5] [5] W. Aussenegg and C. Cech. A New Copula Approach for HighDimensional Real World Portfolios. Working Paper Series Number 68 / 2012, University of Applied Sciences bfi Vienna. (2012).

[6] [6] K. Autchariyapanitkuly, S. Chanaimz, and S. Sriboonchitta. Portfolio Optimization of Stock Returns in High-Dimensions: A Copula-Based Approach. Thai Journal of Mathematics, Special Issue on : Copula Mathematics and Econometrics (2011), p.11-23.

[7] K. Zhu, W. Yamaka, and S. Sriboonchitta. Multi-Asset Portfolio Returns: A Markov Switching Copula-Based Approach. Special Issue on Applied Mathematics: Bayesian Econometrics (2016), p.183-200.

[8] D. B. Nelson. ARCH Models as Diffusion Approximations. Journal of Econometrics, Vol. 45 (1990), p. 7-38.

[9] D. B. Nelson. Conditional Heteroskedasticity in Asset Returns: A New Approach, Econometrica, Vol. 59 (1991), p. 347-370.

[10] M. McAleer and C. Hafner. A One Line Derivation of EGARCH, Econometrics, 2(2) (2014), 92-97.

[11] C-L. Chang and M. McAleer. The Correct Regularity Condition and Interpretation of Asymmetry in EGARCH. TI 2017-056/III, Tinbergen Institute Discussion Paper (2017). 